\title{
Seeing it differently: visual processing in autism
}

\section{Marlene Behrmann, Cibu Thomas and Kate Humphreys}

Department of Psychology and Center for the Neural Basis of Cognition, Carnegie Mellon University, Pittsburgh, Pennsylvania, 15213-3890, USA

Several recent behavioral and neuroimaging studies have documented an impairment in face processing in individuals with Autism Spectrum Disorder (ASD). It remains unknown, however, what underlying mechanism gives rise to this face processing difficulty. One theory suggests that the difficulty derives from a pervasive problem in social interaction and/or motivation. An alternative view proposes that the faceprocessing problem is not entirely social in nature and that a visual perceptual impairment might also contribute. The focus of this review is on this latter, perceptual perspective, documenting the psychological and neural alterations that might account for the face processing impairment. The available evidence suggests that perceptual alterations are present in ASD, independent of social function.

\section{Introduction}

Autism is a neurodevelopmental disorder characterized by impairments in communication and social cognition, co-occurring with repetitive, stereotyped behaviors. Although not considered prosopagnosic per se, individuals with autism, also known as Autism Spectrum Disorder (ASD), often fail to recognize people. Indeed, the last decade has witnessed an explosion of data from behavioral and neuroimaging studies confirming that an impairment in face processing is widespread and present from an early age in ASD [1,2]. Questions about the cognitive and brain mechanisms involved in autism are the subject of intense debate in cognitive science and the issue addressed in this review concerns the underlying source of the face processing impairment. One explanation for the apparent difficulty with faces is that it derives from a pervasive problem in social interaction and the reward and motivational value of social stimuli. An alternative view is that the difficulty with faces is not entirely social in origin but that a visual perceptual impairment also contributes to this difficulty. The focus here is on this latter perspective and on the psychological and neural mechanisms that might give rise to the atypical face processing in ASD.

The face processing difficulty in ASD extends beyond face recognition, affecting the perceptual discrimination of faces too, as evident in same/different judgement tasks [3]

Corresponding author: Behrmann, Marlene (behrmann@cmu.edu) (see Figure 1a and b). In addition, individuals with ASD do not always show the typical superiority for upright over inverted faces ([4,5] but see [6]) and perform atypically on tests of face memory but do well on tests of memory for buildings and leaves [7]. These face processing difficulties are more prevalent among children and cognitively less able individuals, although some studies report a reliable decrement in adults too $[3,8,9]$.

The face processing impairment also extends beyond identity processing to affect processing of emotional expression $[4,10,11]$, possibly independently of IQ. Interestingly, the difficulties in emotional expression processing seem to be exaggerated in the analysis of fear, perhaps because of reduced attention to core features of the face $[10,12]$, particularly the eyes (although see [13]), which are most informative for expressions like fear [11]. Difficulties in discriminating facial gender [14] and unusual patterns of eye gaze processing [15-17] have also been documented in ASD, with the latter perhaps reflecting the failure to track the intentions of the other individual [18].

The decrement in face processing ability is consistent with findings from recent functional imaging studies, documenting weak activation of the fusiform gyrus in ASD in response to faces [19-23], with concurrent activation of a host of other cortical regions not usually associated with face processing [19,21-25]. Other regions of the 'social brain network', including the superior temporal sulcus and amygdala, are also functionally atypical in ASD individuals [15,26-28]. The neuroimaging findings, however, are controversial: some studies do, in fact, report fusiform activation in ASD [29,30] with greater signal for familiar than unfamiliar faces [30], as typically expected (see [31] for discussion of the discrepant results).

Abnormal neural responses are also observed in ASD in ERP studies: the N170 component, the best known ERP marker for faces [32-34], is reduced or less specific and its scalp topography is atypical, with more bilateral than unilateral (right) distribution (see also MEG study [35]). Also, atypically [1], the N170 in ASD is not delayed for inverted relative to upright faces [32] and later components (P400; Nc) do not differentiate between familiar and unfamiliar faces [1].

The hypoactivation of the face-selective cortical regions may not be attributable to a fundamental failure to engage the fusiform gyrus; fusiform activation was obtained in a child who was a Digimon expert when viewing Digimon 
(a)
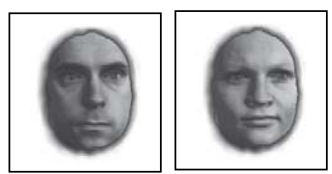

Different

gender
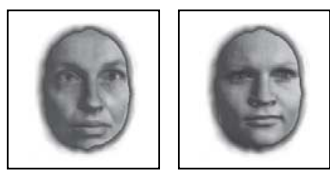

Different

individual (same gender)

(b)

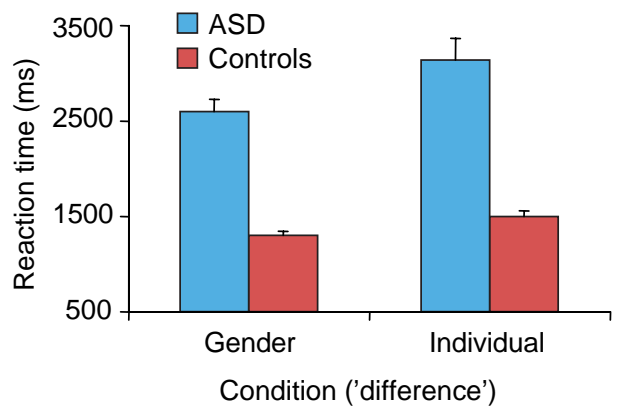

(c)
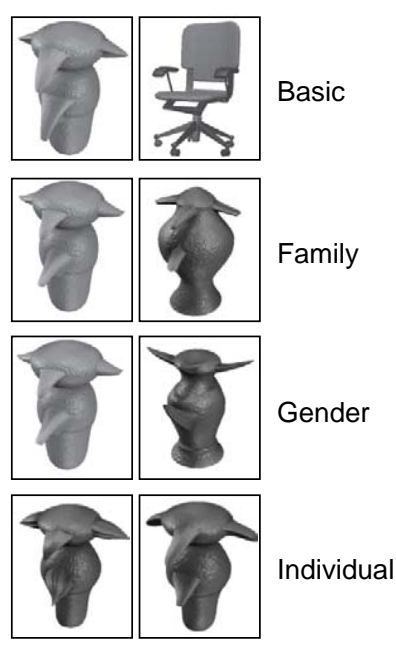

(d)

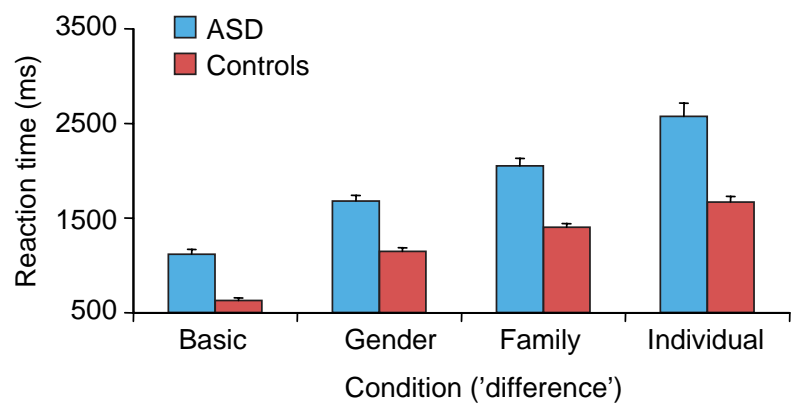

TRENDS in Cognitive Sciences

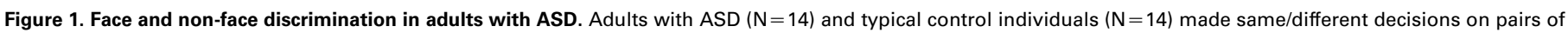

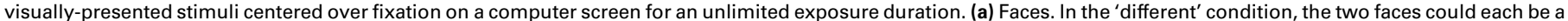

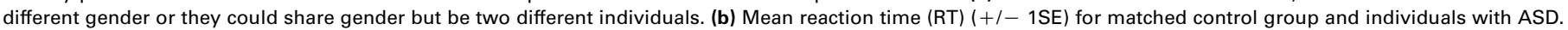

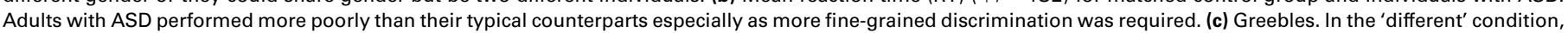
the two images could differ at a 'basic' level (Greebles vs. chair), at a 'family' level (different main body parts), at a 'gender' level (appendages oriented up vs. down) or at an

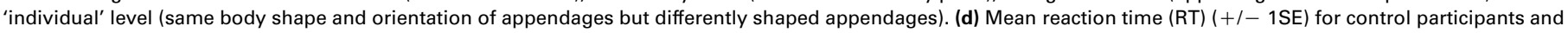

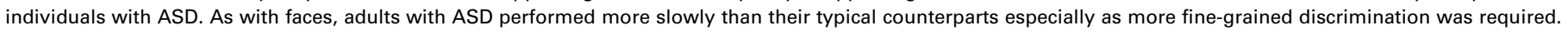
Reprinted with permission from Ref. [3].

(but not faces) in the scanner [23]. Rather, the hypoactivation may reflect the failure to attend to the eye region $[16,29]$, the failure to engage in configural processing and/ or the failure to engage the fusiform region for face processing during the course of maturation and experience (see below).

\section{Is the deficit in face processing perceptual in nature?}

In spite of the data documenting atypical face processing in ASD, there is surprisingly little consensus concerning the source of this impairment. Several explanations, which are not necessarily mutually exclusive, have been offered. The first is that the difficulty with faces is secondary to a primary impairment in social motivation or affective tagging of socially relevant stimuli [1,2]: faces are not intrinsically emotionally salient (and may even be aversive) to individuals with ASD, perhaps because of atypical amygdala development. Consequently, individuals with ASD do not look at faces much [31] and not at the eyes specifically [16]. The social deficit might also arise from a general impairment in empathizing and theory of mind (ToM) [28], and from its precursors, deficits in joint attention and imitation. A second possibility is that face processing in ASD is not deviant per se but is simply developmentally delayed and the lack of functional specialization in the ASD behavioral and neural data reflects a pattern more akin to that seen in younger children [36,37]. Yet a third explanation (and the focus of this review) is that a more basic perceptual difficulty might play a significant, if not primary, contributing role to the face difficulties $[3,38]$ in ASD. We examine the data supporting this last interpretation next.

\section{Is there a primary perceptual deficit in individuals with ASD?}

The argument for a perceptual alteration, which may underlie the face processing impairment, is based on the observation that individuals with ASD are particularly attentive to local details or featural information and, concurrently, may fail to extract the gist or gestalt of the input. In fact, Kanner's original description of autism included 'the inability to experience wholes without full attention to the constituent parts', and 'a persistent preoccupation with parts of objects' is one of the diagnostic 


\section{Box 1. Are there perceptual alterations in modalities other than vision?}

Strong affinities and aversions for various tactile sensations, smells, flavors and sounds are often reported by individuals with ASD or by their parents [67], and claims of sensory dominance, over-arousal or under-arousal have long been associated with ASD. Despite early interest in these issues, recent empirical evidence to support these sensory alterations in ASD is limited [62,68].

Recent studies on auditory sensitivity in ASD have yielded somewhat mixed findings: some find hypo-responsiveness to auditory stimuli [69], others find no differences relative to a comparison group [70], and still others report superior pitch sensitivity [71], akin to reports of enhanced visual processing [39,54]. High-functioning children with ASD apparently demonstrate superior memory for pitch, labeling and disembedding pre-exposed tones from musical chords relative to controls [72]. Other psychoacoustic studies suggest that ASD individuals have poor frequency selectivity abilities, potentially accounting for the difficulty they have understanding speech amidst background noise [43]. There are also striking parallels to findings from global/local tasks in the visual domain. Adults with ASD perform well (and sometimes better than their comparison group) on tasks involving simple operations [73], such as matching changes of pitch direction at the local level [74] and judging pairs of melodies at the local level [75]. In this latter study, the ASD group did

criteria for autistic disorder (DSM-IV, APA, 1994). The enhancement of featural or detail-focused processing [39], sometimes conceptualized as a cognitive processing style or bias [40,41], has been referred to as 'weak central coherence' [42] and many studies attest to the local bias in ASD (for comprehensive review, see [41]). The enhanced local bias may have far-reaching effects, including fragmented and super-acute perception [39] and reduced generalization [43], and it extends beyond vision (see Box 1), implicating a more widespread multimodal difficulty.

Weak central coherence $[41,43,44]$ is characterized by the superior performance of ASD individuals, relative to their peers, under conditions in which the processing of details is required as in Block Design, finding embedded figures, visual search and learning highly confusable patterns [39,41,45] (see Figure 2). Enhanced processing of details comes with a price, however, and ASD individuals often, although not always $[45,46]$, perform poorly in tasks where grouping is required [47]. Additionally, ASD individuals might identify the local letter of hierarchical stimuli better than the global letter (e.g. the ' $\mathrm{H}$ ' in a display of a large ' $\mathrm{S}$ ' made up from small ' $\mathrm{H}$ 's; they see the 'trees before the forest'), in contrast to the typical global advantage ('forest before the trees'), and sometimes not show an impairment in discriminating the melodies at the global level. Tasks involving more complex auditory operations such as stimulus evaluation are typically performed poorly and generate inferior ERP and brain activation in ASD individuals [73].

There are relatively few recent studies on tactile sensation, gustation and olfaction in ASD. In one study, using parental report on the Short Sensory Profile [76], children with ASD and Fragile-X syndrome (FXS) showed more sensory symptoms in tactile sensitivity and auditory filtering than groups of matched children with developmental disabilities and typically developing children. The ASD group, however, demonstrated greater abnormal response to taste and smell than any other group.

One possible reason for the variability in findings of sensory and perceptual function in ASD is the use of different experimental methods. It is also possible that perceptual atypicalities arise not only from enhanced perceptual function (and overfunctioning brain regions) [62] but also from integration and organization across different sensory modalities [77]. Clearly, systematic empirical work involving investigations within and between multiple modalities is required to shed light on the atypical sensory symptoms in ASD and the relation between these and other behavioral symptoms.

even show interference from the local letter when identifying the global letter $[48,49]$. Although there are conflicting results from studies using hierarchical displays $[38,41,50]$, in one recent study, adults with ASD showed both the local advantage as well as local-to-global interference [3]. This disproportionate appeal of local details in ASD has been likened to a deficit in expanding the attentional 'window' [51]. It is the case, however, that attentional cueing in ASD can lead to configural-based processing of faces [52], suggesting that the local bias is not the only processing mode available (although it might be the default) and that more holistic processing is also possible. Whether the detail-focused local enhancement necessarily co-occurs with poor global processing or whether they are dissociable remains the subject of ongoing controversy [39]. Neurobiological markers and the possible reduction of connectivity [53] normally required for integrating local elements [54] are being explored as possible neural sources of the local bias.

\section{The perceptual deficit in relation to face processing}

Although not all studies consistently uncover a perceptual deficit in ASD, there is growing consensus that perceptual alterations may well be characteristic of autism [39], independent of deficits in executive function, social (a)

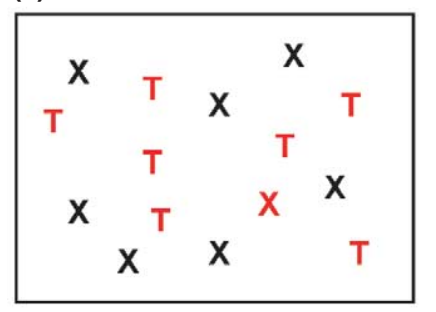

(b)

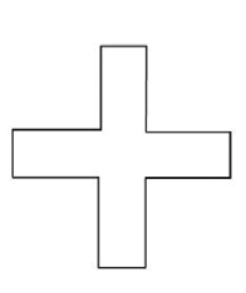

(c)

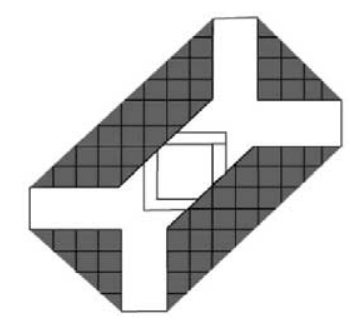

(c)

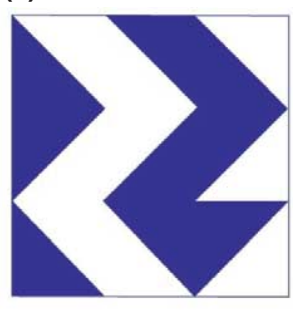

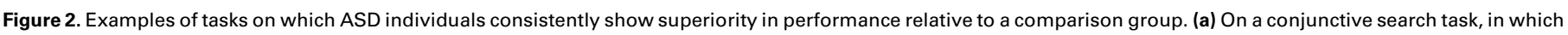

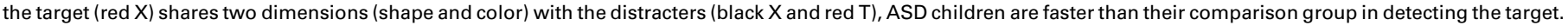

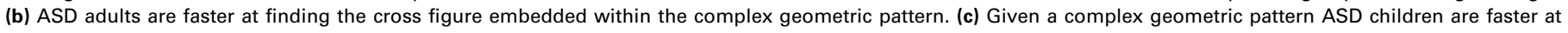
recreating this pattern by assembling a set of blocks composed of similar color surfaces. 
behavior and ToM [41]. How might this perceptual bias towards local elements affect face processing? It has been suggested that because faces are perceptually similar, part or feature-based processing is not sufficient for identification, so face processing is particularly reliant on configural processing. Moreover, the need for configural processing and deriving the spatial relations between the local elements is especially crucial for identifying individual instances or exemplars. Faces, unlike other objects, are typically identified at the individual level and maximally fine-grained representations are required under these conditions. The upshot of this is that the undue focus on local stimulus aspects in ASD puts face processing at greatest risk.

The impairment for faces in ASD might then result from the tendency to represent and encode visual information locally, on a part-by-part basis rather than holistically (and ASD individuals might even focus on less optimal parts, see above). Additionally, the fact that individuals with ASD show a reduced or absent 'faceinversion effect' (although see [6]) suggests that they rely less on 'configural' or 'holistic' processing. Also, when the need for holistic information in faces is increased, adults with ASD perform disproportionately poorly: not only is the time to discriminate between two novel faces [3] slow but it becomes slower as the discrimination is more finegrained (e.g. differentiating between two faces of the same gender but different identities is harder than between two faces of different genders). Interestingly, this slowing correlates with the extent of the local advantage in a hierarchical letter task [3] (for similar correlation between reduced global responding and emotion recognition in children with autism, see [55]). Although correlation is not causation, this does suggest a possible relationship between a perceptual bias to local elements and impaired face processing. Adolescents with ASD also show a reduced 'face composite effect' [4] and children with ASD show a reduced whole-face advantage for eyes and noses (although not for mouths) [56], both reflecting a decreased reliance on holistic processing. This reduced whole-face advantage, however, can be normalized when the individuals are cued with the face part on which to base their decision (e.g. instructed to 'Look at the eyes') [52]. Again, this suggests that holistic processing is not impossible for this population, but that it might not be their default processing style.

\section{Does the perceptual deficit extend to other classes of visual stimuli?}

One obvious prediction from the claim that there is a general perceptual deficit in ASD is that an impairment in processing stimuli other than faces should also be observed, although perhaps to a lesser extent given the undue reliance on relational processing for faces. Non-face processing is rather understudied in ASD and to the extent that it has, it is usually in the context of using nonface inputs as control stimuli for faces. Some studies of non-face processing claim that individuals with ASD do not show deficits on complex object perception tasks [31]. However, recognition memory for stimuli such as motorbikes, cats and horses is poorer in ASD than in the typical comparison group [7]. Also, adults with ASD discriminate non-face objects (Greebles and also common everyday objects; see Figure 1c and d) more slowly than their comparison group [3] and evince disproportionate slowing as more fine-grained differentiation is required; deciding that two different instances of a duck (exemplar or individual level) are 'different' and that a chair and a duck are 'different' (basic level) takes significantly more time for the ASD than control group. Additionally, there is a correlation between the ability to make fine-grained discriminations and the local advantage, as reflected in performance on a hierarchical letter task. These findings suggest that ASD individuals may be impaired (or biased) to process visual information at a more local level and that the failure to derive more holistic representations is particularly disadvantageous when similar perceptual exemplars (individual level) must be differentiated, be they faces or not.

Neuroimaging data examining the cortical substrate of object processing in ASD have also produced mixed results. Although MEG has revealed greater than normal variation in the location of the sources for ASD than for controls in face and non-face tasks [35], ERPs for young children viewing familiar and unfamiliar toys show patterns quite similar to those of controls [1]. A functional MRI study of subordinate object discrimination using cars, bottles, birds, planes and chairs revealed no consistent differences between the ASD and control groups, although only two regions of interest, the inferior temporal and fusiform gyri bilaterally, were examined [19].

It is still the case, however, that faces seem to be treated more like objects: for example, the reduced inversion effect sometimes seen for faces in ASD and the reduced holistic processing of faces in ASD [56] are more akin to the profile associated with object processing in typical individuals. Further, some imaging studies indicate that face processing may also lead to activation of brain regions more typically associated with object processing [19] or with visual search, suggesting that the individuals with ASD may be performing a more object-related feature-based search of the faces [22] rather than the expected configural processing engaging the fusiform region.

Is there a more general underlying perceptual difficulty? Before concluding that the face and non-face difficulty perceptual decrement results from the local bias (and holistic/configural difficulty) in ASD, we need to examine whether an even lower-level visual processing deficit may be ultimately responsible for the perceptual profiles in ASD. Global, holistic properties of a display are thought to be processed mainly by low spatial frequency channels, a property of the magnocellular system, and the local elements by higher-spatial frequency channels, a property of the parvocellular system. If the magnocellular system is affected in ASD, global properties of faces and objects may not be well perceived by virtue of a restriction on low spatial frequency information and ASD individuals may show an increased reliance on high spatial frequency information [57]. Few studies, however, have measured the spatial frequency thresholds of individuals with ASD but one that did reports normal log contrast thresholds 
across the range from low ( $1 \mathrm{cpi}$ ) to high (30 cpi) frequency [3]. Consistent with a possible magnocellular source, however, several studies have reported that individuals with ASD are less sensitive to visual motion [58,59], another assumed property of the magnocellular system, and require higher levels of motion coherence to discriminate the direction of moving dots in a display $[60,61]$.

There does not seem to be sufficient evidence to support the notion of a primary impairment in the magnocellular pathway: two recent studies showed that first-order visual motion processing was intact in the ASD group relative to a comparison group [44], ruling out a fundamental motion deficit, and showed that only higher-order motion processing was impaired (also [59]). Rather, the elevated motion coherence thresholds and problems in discerning higherorder motion in ASD may arise from the same enhanced local processing and/or reduced holistic processing rather than from a magnocellular restriction (see also [54] for data from a flicker contrast sensitivity test ruling out a magnocellular problem): to determine the direction or pattern of motion in the display requires that the observer derive the relations between the local elements, and it is thus configural processing, rather than motion perception, that is affected.

Others have also suggested a low-level visual source for the perceptual alterations in ASD but here the claim is that the perceptual pattern in ASD arises from superior or enhanced perceptual ability, with increased sensitivity to locally oriented processing attributable to the overfunctioning of early visual cortex $[39,62]$. Some evidence to support this is a recent study in which individuals with autism show at least normal (and possibly superior) ability in identifying the orientation of simple, luminance-defined (or first-order) gratings [54]. There have, however, been remarkably few detailed investigations of the functional organization of the early visual system in individuals with ASD. One recent fMRI study found no difference in the retinotopic maps in a group of adults with ASD and their typical counterparts [63]. Regional glucose metabolism is apparently increased [64] in early visual cortex but whether this indicates superior or inferior function remains unclear. Clearly, further investigations of lower-level visual function are urgently needed.

\section{Reconciliation?}

This review has explored the possible contribution of altered perceptual function to the impairment in face processing abilities in individuals with ASD. The data, indicating a local bias and perhaps an even lower-level perceptual alteration, clearly indicate a difference in perceptual function in both children and adults with ASD. Taken together, these data suggest that the impairment in face processing need not necessarily arise solely from a social and/or motivational source but that a perceptual impairment might also contribute to the difficulty with face processing. Of course the social and perceptual explanations need not be mutually exclusive and both might contribute, perhaps even interactively, to the difficulties in face processing (for computational account of this interaction, see [65]). One potentially persuasive reconciliation between the perceptual and social explanations proposes that it is the lack of expertise or familiarity with faces that reduces the functional specialization of face-selective cortical areas. The lack of expertise itself is a function of reduced social abilities and the decreased social orienting results in failure to develop the requisite behavioral and neural mechanisms ultimately needed for face expertise $[2,66]$. Because experience drives cortical specialization [37], reduced attention to and interest in faces would be reflected in decreased cortical specialization and abnormal brain circuitry for face processing. Expertise in other visual domains may still engage the fusiform gyrus [23] and interventions targeted at developing face expertise in young children may help in configuring the underlying behavioral and neural circuitry.

Although compelling, this rapprochement may not suffice fully as some alterations in perception in ASD do not easily fit this account. Whether the full range of perceptual alterations in ASD arise from a local bias and/or poor global processing, from just one of these or from enhanced, superior low-level visual functioning remains to be explored. Moreover, the perceptual changes in non-face as well as face processing must be accounted for as well as the correlation between object processing and the local bias and how this might be reconciled with the interaction of social and perceptual factors is not obvious. The ultimate explanation must also account for

\section{Box 2. Questions for future research}

- Are the various difficulties observed in face processing in individuals with ASD (failure to attend to the eyes; reduced holistic/configural processing; difficulties with identity, expressions, gender, eye gaze) part of the same underlying problem or are they independent and dissociable [78]?

- To what extent is non-face object recognition adversely affected in ASD? Is there a clear neural explanation for this?

- Are there observable alterations in cortical functioning in early parts of the visual system that might definitively explain the local bias [39]?

- What is the source of the variability in behavior and in neural data observed in individuals with ASD? Is ASD really a continuum or are there several distinct disorders with somewhat similar symptomatologies? To what extent do all individuals with ASD show the perceptual deficits and to what extent are these difficulties evidence in both adults and children with ASD? Are the individual differences in brain activation a function of greater variability in brain morphology than is evident in typical populations?

- How do the apparent visual difficulties relate to theories of brain abnormalities in ASD? For example, some theories suggest that there is underconnectivity and lack of synchronization between different cortical regions. Might this account for the failure to integrate disparate aspects of visual information into a coherent whole?

- To what extent are these visual perceptual difficulties, reflected in the failure to bind information into composite whole, observed in other sensory modalities?

- Given that a focus on parts of objects is part of the diagnosis for ASD (Category 3 behaviors: repetitive interests and preoccupations), would global vs. local processing be correlated with these behaviors?

- Are there potential implications for retraining or intervention that might be revealed by studies of the visual system in ASD? 
changes in perception in non-visual modalities (Box 1). Much research remains to be done (see Box 2). The current bottom line, however, is that we probably need to see autism differently and that perceptual alterations are present in ASD, independent of social function.

\section{Acknowledgements}

The writing of this review was supported by grants from NICHD/NIDCD PO1/U19 (PI: Nancy Minshew), which is part of the NICHD/NIDCD Collaborative Programs for Excellence in ASD, The National Alliance for Autism Research and the Cure Autism Now foundation. We thank Drs N. Minshew, B. Luna and L. Mottron for their helpful comments.

\section{References}

1 Dawson, G. et al. (2002) Neural correlates of face and object recognition in young children with autism spectrum disorder, developmental delay, and typical development. Child Dev. 73, 700-717

2 Dawson, G. et al. (2005) Understanding the nature of face processing impairment in autism: insights from behavioral and electrophysiological studies. Dev. Neuropsychol. 27, 403-424

3 Behrmann, M. et al. (2006) Configural processing in autism and its relationship to face processing. Neuropsychologia 44, 110-129

4 Teunisse, J. and De Gelder, B. (2003) Face processing in adolescents with autistic disorder: The inversion and composite effects. Brain Cogn. 52, 285-294

5 van der Geest, J.N. et al. (2002) Gaze behavior of children with pervasive developmental disorder toward human faces: A fixation time study. J. Child Psychol. Psychiatry 43, 669-678

6 Lahaie, A. et al. (2006) Face perception in high-functioning autistic adults: Evidence for superior processing of face parts, not a configural face-processing deficit. Neuropsychology 20, 30-41

7 Blair, R.J. et al. (2002) Fractionation of visual memory: agency detection and its impairment in autism. Neuropsychologia 40, 108-118

8 Barton, J.J. et al. (2004) Are patients with social developmental disorders prosopagnosic? Perceptual heterogeneity in the Asperger and socio-emotional processing disorders. Brain 127, 1706-1716

9 Jemel, B. et al. (2006) Impaired face processing in autism: Fact or artifact? J. Autism Dev. Disord. 36, 91-106

10 Pelphrey, K.A. et al. (2002) Visual scanning of faces in autism. J. Autism Dev. Disord. 32, 249-261

11 Gross, T.F. (2004) The perception of four basic emotions in human and nonhuman faces by children with autism and other developmental disabilities. J. Abnorm. Child Psychol. 32, 469-480

12 Klin, A. et al. (2002) Visual fixation patterns during viewing of naturalistic social situations as predictors of social competence in individuals with autism. Arch. Gen. Psychiatry 59, 809-816

13 Bar-Haim, Y. et al. (2006) Attention to eyes and mouth in highfunctioning children with autism. J. Autism Dev. Disord. 36, 1-7

14 Njiokiktjien, C. et al. (2001) Disordered recognition of facial identity and emotions in three Asperger type autists. Eur. Child Adolesc. Psychiatry 10, 79-90

15 Pelphrey, K.A. et al. (2005) Neural basis of eye gaze processing deficits in autism. Brain 128, 1038-1048

16 Dalton, K.M. et al. (2005) Gaze fixation and the neural circuitry of face processing in autism. Nat. Neurosci. 8, 519-526

17 Senju, A. et al. (2004) Reflexive orienting in response to eye gaze and an arrow in children with and without autism. J. Child Psychol. Psychiatry 45, 445-458

18 Ristic, J. et al. (2005) Eyes are special but not for everyone: the case of autism. Brain Res. Cogn. Brain Res. 24, 715-718

19 Schultz, R.T. et al. (2000) Abnormal ventral temporal cortical activity during face discrimination among individuals with autism and Asperger syndrome. Arch. Gen. Psychiatry 57, 331-340

20 Critchley, H.D. et al. (2000) The functional neuroanatomy of social behaviour: Changes in cerebral blood flow when people with autistic disorder process facial expressions. Brain 123, 2203-2212

21 Pierce, K. et al. (2001) Face processing occurs outside the fusiform 'face area' in autism: evidence from functional MRI. Brain 124, 2059-2073
$22 \mathrm{Hubl}$, D. et al. (2003) Functional imbalance of visual pathways indicates alternative face processing strategies in autism. Neurology $61,1232-1237$

23 Grelotti, D.J. et al. (2005) fMRI activation of the fusiform gyrus and amygdala to cartoon characters but not to faces in a boy with autism. Neuropsychologia 43, 373-385

24 Schultz, R.T. et al. (2003) The role of the fusiform face area in social cognition: implications for the pathobiology of autism. Philos. Trans. R. Soc. Lond. B Biol. Sci. 358, 415-427

25 Wang, A.T. et al. (2004) Neural correlates of facial affect processing in children and adolescents with autism spectrum disorder. J. Am. Acad. Child Adolesc. Psychiatry 43, 481-490

26 Castelli, F. et al. (2002) Autism, Asperger syndrome and brain mechanisms for the attribution of mental states to animated shapes. Brain 125, 1839-1849

27 Bachevalier, J. and Loveland, K.A. (2006) The orbitofrontal-amygdala circuit and self-regulation of social-emotional behavior in autism. Neurosci. Biobehav. Rev. 30, 97-117

28 Baron-Cohen, S. and Belmonte, M.K. (2005) Autism: a window onto the development of the social and the analytic brain. Annu. Rev. Neurosci. 28, 109-126

29 Hadjikhani, N. et al. (2004) Activation of the fusiform gyrus when individuals with autism spectrum disorder view faces. Neuroimage 22 , $1141-1150$

30 Pierce, K. et al. (2004) The brain response to personally familiar faces in autism: findings of fusiform activity and beyond. Brain 127, $2703-2716$

31 Schultz, R.T. (2005) Developmental deficits in social perception in autism: the role of the amygdala and fusiform face area. Int. J. Dev. Neurosci. 23, 125-141

32 McPartland, J. et al. (2004) Event-related brain potentials reveal anomalies in temporal processing of faces in autism spectrum disorder. J. Child Psychol. Psychiatry 45, 1235-1245

33 Dawson, G. et al. (2005) Neurocognitive and electrophysiological evidence of altered face processing in parents of children with autism: Implications for a model of abnormal development of social brain circuitry in autism. Dev. Psychopathol. 17, 679-697

34 O'Connor, K. et al. (2005) The neurophysiological correlates of face processing in adults and children with Asperger's syndrome. Brain Cogn. 59, 82-95

35 Bailey, A.J. et al. (2005) Abnormal activation of face processing systems at early and intermediate latency in individuals with autism spectrum disorder: a magnetoencephalographic study. Eur. J. Neurosci. 21, 2575-2585

36 Grice, S.J. et al. (2005) Neural correlates of eye-gaze detection in young children with autism. Cortex 41, 342-353

37 Johnson, M.H. et al. (2005) The emergence of the social brain network: Evidence from typical and atypical development. Dev. Psychopathol. $17,599-619$

38 Dakin, S. and Frith, U. (2005) Vagaries of visual perception in autism. Neuron 48, 497-507

39 Caron, M-J. et al. Cognitive mechanisms, specificity and neural underpinnings of the block design peak in autism. Brain (in press)

40 Iarocci, G. et al. (2006) Global-Local visual processing in high functioning children with autism: Structural vs. implicit task biases. J. Autism Dev. Disord. 36, 117-129

41 Happé, F.G.E. and Frith, U. (2006) The weak coherence account: Detail-focused cognitive style in autism spectrum disorders. J. Autism Dev. Disord. 36, 5-25

42 Frith, U. and Happe, F. (2005) Autism spectrum disorder. Curr. Biol. 15, R786-R790

43 Plaisted, K. et al. (2003) Towards an understanding of the mechanisms of weak central coherence effects: experiments in visual configural learning and auditory perception. Philos. Trans. R. Soc. Lond. B Biol. Sci. 358, 375-386

44 Pellicano, E. et al. (2005) Abnormal global processing along the dorsal visual pathway in autism: a possible mechanism for weak visuospatial coherence? Neuropsychologia 43, 1044-1053

45 Edgin, J.O. and Pennington, B.F. (2005) Spatial cognition in Autism Spectrum Disorders: Superior, impaired, or just intact? J. Autism Dev. Disord. 35, 729-745 
46 Rouse, H. et al. (2004) Do children with autism perceive second-order relational features? The case of the Thatcher illusion. J. Child Psychol. Psychiatry 45, 1246-1257

47 Brosnan, M.J. et al. (2004) Gestalt processing in autism: failure to process perceptual relationships and the implications for contextual understanding. J. Child Psychol. Psychiatry 45, 459-469

48 Plaisted, K. et al. (1999) Children with autism show local precedence in a divided attention task and global precedence in a selective attention task. J. Child Psychol. Psychiatry 40, 733-742

49 Rinehart, N. et al. (2000) Atypical interference of local detail on global processing in high-functioning autism and Asperger's disorders. J. Child Psychol. Psychiatry 41, 769-788

50 Mottron, L. et al. (2003) Locally oriented perception with intact global processing among adolescents with high-functioning autism: evidence from multiple paradigms. J. Child Psychol. Psychiatry 44, 904-913

51 Mann, T.A. and Walker, P. (2003) Autism and a deficit in broadening the spread of visual attention. J. Child Psychol. Psychiatry 44, 274-284

52 Lopez, B. et al. (2004) Face processing in high-functioning adolescents with autism: Evidence for weak central coherence. Vis. Cogn. 11, 673-688

53 Just, M.A. et al. (2004) Cortical activation and synchronization during sentence comprehension in high-functioning autism: evidence of underconnectivity. Brain 127, 1811-1821

54 Bertone, A. et al. (2005) Enhanced and diminished visuo-spatial information processing in autism depends on stimulus complexity. Brain 128, 2430-2441

55 Gross, T.F. (2005) Global-local precedence in the perception of facial age and emotional expression by children with autism and other developmental disabilities. J. Autism Dev. Disord. 35, 773-785

56 Joseph, R.M. and Tanaka, J. (2003) Holistic and part-based face recognition in children with autism. J. Child Psychol. Psychiatry 44, 529-542

57 Deruelle, C. et al. (2004) Spatial frequency and face processing in children with autism and Asperger syndrome. J. Autism Dev. Disord. $34,199-210$

58 Gepner, B. and Mestre, D. (2002) Rapid visual-motion integration deficit in autism. Trends Cogn. Sci. 6, 455

59 Blake, R. et al. (2003) Visual recognition of biological motion is impaired in children with autism. Psychol. Sci. 14, 151-157

60 Milne, E. et al. (2002) High motion coherence thresholds in children with autism. J. Child Psychol. Psychiatry 43, 255-263

61 Spencer, J. et al. (2000) Motion processing in autism: evidence for a dorsal stream deficiency. Neuroreport 11, 2765-2767
62 Mottron, L. et al. (2006) Enhanced perceptual functioning in autism: An update and eight principles of autistic perception. J. Autism Dev. Disord. 36, 27-43

63 Hadjikhani, N. et al. (2004) Early visual cortex organization in autism: An fMRI study. Neuroreport 15, 267-270

64 Hazlett, E.A. et al. (2004) Regional glucose metabolism within cortical Brodmann areas in healthy individuals and autistic patients. Neuropsychobiology 49, 115-125

65 Grossberg, S. and Seidman, D. Neural dynamics of autistic behaviors. Psychol. Rev. (in press)

66 Grelotti, D.J. et al. (2002) Social interest and the development of cortical face specialization: what autism teaches us about face processing. Dev. Psychobiol. 40, 213-225

67 Jones, R.S.P. et al. (2003) First-hand accounts of sensory perceptual experiences in autism: A qualitative analysis. J. Intellect. Dev. Disabil. 28, 112-121

68 Rogers, S.J. and Ozonoff, S. (2005) Annotation: what do we know about sensory dysfunction in autism? A critical review of the empirical evidence. J. Child Psychol. Psychiatry 46, 1255-1268

69 Tecchio, F. et al. (2003) Auditory sensory processing in autism: a magnetoencephalographic study. Biol. Psychiatry 54, 647-654

70 Ceponiene, R. et al. (2003) Speech-sound-selective auditory impairment in children with autism: they can perceive but do not attend. Proc. Natl. Acad. Sci. U. S. A. 100, 5567-5572

71 Bonnel, A. et al. (2003) Enhanced pitch sensitivity in individuals with autism: a signal detection analysis. J. Cogn. Neurosci. 15, 226-235

72 Heaton, P. (2003) Pitch memory, labelling and disembedding in autism. J. Child Psychol. Psychiatry 44, 543-551

73 Samson, F. et al. (2006) Can spectro-temporal complexity explain the autistic pattern of performance on auditory tasks? J. Autism Dev. Disord. 36, 65-76

74 Foxton, J.M. et al. (2003) Absence of auditory 'global interference' in autism. Brain 126, 2703-2709

75 Mottron, L. et al. (2000) Local and global processing of music in highfunctioning persons with autism: beyond central coherence? J. Child Psychol. Psychiatry 41, 1057-1065

76 Rogers, S.J. et al. (2003) Parent reports of sensory symptoms in toddlers with autism and those with other developmental disorders. J. Autism Dev. Disord. 33, 631-642

77 Iarocci, G. and McDonald, J. (2006) Sensory integration and the perceptual experience of persons with autism. J. Autism Dev. Disord. $36,77-90$

78 Hefter, R.L. et al. (2005) Perception of facial expression and facial identity in subjects with social developmental disorders. Neurology 65 , $1620-1625$

\section{The ScienceDirect collection}

ScienceDirect's extensive and unique full-text collection covers more than 1900 journals, including titles such as The Lancet, Cell, Tetrahedron and the full suite of Trends, Current Opinion and Drug Discovery Today journals. With ScienceDirect, the research process is enhanced with unsurpassed searching and linking functionality, all on a single, intuitive interface.

The rapid growth of the ScienceDirect collection is a result of the integration of several prestigious publications and the ongoing addition to the Backfiles - heritage collections in a number of disciplines. The latest step in this ambitious project to digitize all of Elsevier's journals back to volume one, issue one, is the addition of the highly cited Cell Press journal collection on ScienceDirect. Also available online for the first time are six Cell titles' long-awaited Backfiles, containing more than 12,000 articles that highlight important historic developments in the field of life sciences.

\section{For more information, visit www.sciencedirect.com}

